\title{
Preface
}

\section{Managing Controversial \\ Issues in Thoracic Surgery}

(D) CrossMark

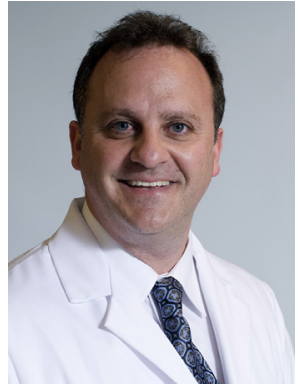

Michael Lanuti, MD, FACS

Editor

This issue of Thoracic Surgery Clinics depicts the contemporary controversies around the management of both benign and malignant conditions in Thoracic Surgery. The discussions are very relevant to the daily practice of surgeons and provide an organized summary and interpretation of the latest published literature relevant to each topic. Many issues in our field remain controversial by virtue of a paucity of prospective, randomized clinical trials, which assess the latest advance in treatments (ie, stereotactic body radiation therapy for stage I non-small cell lung cancer [NSCLC], approach to oligometastatic NSCLC, multimodality treatment of stage IIIA NSCLC, management of locally advanced thymoma, efficacy of lymph node dissection in pulmonary metastectomy) or surgical innovations (endobronchial ultrasound in staging lung cancer, benefit of surgical plication of a paralyzed diaphragm, stenting esophageal perforations, or efficacy of sympathectomy for focal hyperhidrosis). These areas of controversy are not necessarily comprehensive reviews of the literature, but a working prospective on each issue that may provide additional clarity. I thank the contributing authors for their valuable time and expertise in summarizing these specific areas of controversy.

Michael Lanuti, MD, FACS Associate Professor of Surgery Harvard Medical School Director of Thoracic Oncology Division of Thoracic Surgery Massachusetts General Hospital 55 Fruit Street, Blake 1570 Boston, MA 02114, USA

E-mail address: mlanuti@mgh.harvard.edu 